\title{
Interactions between physical activity and diet in the regulation of body weight
}

\author{
Melanie S. Moore \\ MRC Human Nutrition Research, Downham's Lane, Cambridge, CB4 IXJ, UK
}

\begin{abstract}
The increasing worldwide prevalence of obesity suggests that there is a chronic positive energy balance. This situation implies poor coupling between energy intake and energy expenditure, but the contribution of each of these factors remains unclear. Epidemiological data suggests that physical activity has a role in the prevention of weight gain, although there is little evidence that it has a beneficial role in weight loss. High-fat diets have also been implicated, evidence from a variety of sources suggests that diets high in fat undermine appetite regulatory mechanisms. There has been much research to investigate the coupling between energy expenditure and energy intake in the short term in an attempt to elucidate some of the mechanisms involved. However, mechanisms regulating appetite are very complex, and it is currently unclear at which points physical activity and diet may have an influence.
\end{abstract}

Obesity: Physical activity: High fat diet

The present review will discuss some of the evidence linking the effects of physical activity and diet, and especially their interactions, in the regulation of body weight. This problem is particularly pressing in the face of a continued rise in the prevalence of obesity. Recent data from the Health Survey for England (Prescott-Clarke \& Primatesta, 1999) showed that one in five women and approximately one in six men were obese $\left(\mathrm{BMI}>30 \mathrm{~kg} / \mathrm{m}^{2}\right)$. More alarmingly, $53 \%$ of the women and $62 \%$ of the men were overweight $\left(\mathrm{BMI}>25 \mathrm{~kg} / \mathrm{m}^{2}\right)$. The prevalence of obesity has more than doubled since 1980, and the trend shows no indication of slowing down, suggesting there is a chronic positive energy balance. It has been estimated that between 5 and $10 \%$ of the worldwide adult population are now obese (Seidell, 1999), and even in lower-income countries where obesity used to be rare it is becoming more common (Popkin \& Doak, 1998). For example, in Brazil the prevalence of obesity almost doubled over the 15-year period between 1974-5 and 1989 (Monteiro et al. 1995).

Ecological data indicates a strong relationship between increases in proxy measures of inactivity, such as television viewing or car ownership, and obesity (Prentice \& Jebb, 1995), suggesting that the increasingly sedentary habits associated with modern lifestyles play an important role in the development of obesity. Conversely, there appears to be little or no relationship between obesity and actual levels of reported energy intake, which have fallen since the 1970s, concurrent with the rising obesity prevalence (Ministry of Agriculture Fisheries and Foods, 1991). Similar effects have been observed in the USA (Weisnier et al. 1998). It is evident, however, that not all sedentary individuals are overweight, and indeed many lean individuals are very inactive. This inter-individual variability is due to differences in the coupling between energy intake and energy expenditure. Thus, individuals who have successfully down regulated their levels of energy intake to match their low levels of expenditure may remain lean despite a sedentary lifestyle. However, this process appears not to have occurred at the population level, suggesting a poor coupling between energy intake and energy expenditure.

\section{Regulation of energy intake}

Understanding the physiological processes which regulate body weight has long been the focus of much research in many different areas of science, including nutrition, physiology, epidemiology, psychology and cellular biology. Early hypotheses described single factors to explain the control of food intake, e.g. the Mayer (1953) glucostatic hypothesis and the Kennedy (1953) lipostatic theory. It is now evident that appetite involves the integrated response of many different factors (Schwartz et al. 1999). Schwartz 
et al. (1999) have recently updated their model of energy homeostasis, which involves the integration of long-term adiposity-related signals such as the hormones leptin and insulin with short-term meal-related signals such as gastrointestinal peptides (e.g. cholecystokinin). Long-term signals are believed to regulate the size of adipose stores through a negative feedback mechanism which acts at the level of the central nervous system (Woods et al. 1979; Campfield et al. 1995). Superimposed on this long-term system is a shorter-acting system, which is independent of adiposity. This system controls the initiation and termination of individual meals and includes gastrointestinal peptides, glucose, behavioural and emotional influences, and effects of diet composition. Levels of the longer-acting hormones, which are determined by the size of adipose stores, may govern the sensitivity of the central nervous system to the shorter-acting mechanisms, e.g. insulin potentiates the action of cholecystokinin (Figlewicz et al. 1986). Physical activity may influence many points in this complex regulatory process, including possible effects on insulin sensitivity, gastrointestinal hormone release and behavioural responses. Effects on substrate balance may also be important. It has been suggested that appetite responses are more sensitive to carbohydrate balance than fat balance, due to the body's greater ability to store fat compared with carbohydrate (Flatt, 1987). Since the intensity and duration of exercise has the ability to alter macronutrient utilization, it may, according to the Flatt (1987) hypothesis, have the ability to alter appetite and hence energy intake.

There is some evidence of an asymmetry in the homeostatic mechanisms which control body weight (Blundell \& King, 1996). Undernutrition appears to provoke stronger feedback mechanisms to restore energy balance than overnutrition, thus suggesting that mechanisms controlling the coupling of energy intake to energy expenditure may vary under different conditions of energy balance. The present review will focus on experimental evidence investigating energy coupling under conditions of energy deficit and energy surplus.

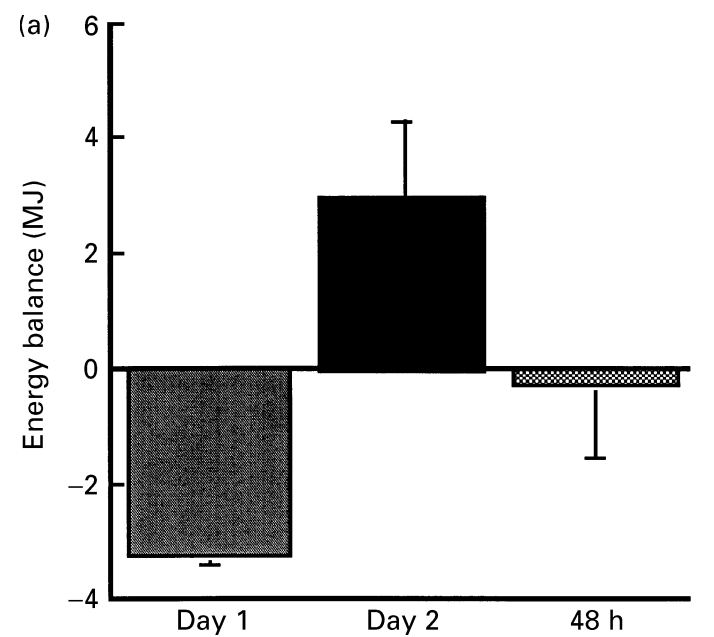

\section{Energy deficits \\ Experimental studies}

Experimental studies have been used to understand factors governing the intake of energy in the short term, and have involved the manipulation of dietary and exercise factors. Goldberg et al. (1998) investigated appetite responses to dietary-induced energy deficits. In this study, conducted within a whole-body calorimeter, nine lean men were put into an energy deficit of approximately $1 \mathrm{MJ}$ on day 1 , achieved either through removal of $1 \mathrm{MJ}$ fat or $1 \mathrm{MJ}$ carbohydrate relative to a control condition. On day 2 subjects ate freely from a diet of medium palatability, which was offered in excess of energy requirements. Subjects successfully compensated on day 2 for the energy deficit created on day 1 with both macronutrient manipulations. There was a very precise coupling between energy intake and expenditure such that $48 \mathrm{~h}$ energy balance was not significantly different from zero with either of the dietary manipulations or between treatments. This finding indicates that in the face of an energy deficit induced through diet there are strong physiological mechanisms to restore energy balance which are not specific to either of the macronutrients tested.

Using a similar protocol and a similar group of subjects, Moore et al. (2000) tested whether a similar response would be observed in the face of an exercise-induced deficit. In this study, also undertaken in a whole-body calorimeter, an exercise deficit was compared with a similar diet-induced deficit. The energy deficit was larger at approximately 3.5 MJ, and was achieved either through removal of a mixed diet or through an increased duration of exercise throughout the day. The $48 \mathrm{~h}$ energy balance with the dietary deficit was, again, not significantly different from zero (Fig. 1(a)). Perhaps surprisingly, there was no differential response to the exercise deficit. Subjects responded to the energy cost of the imposed exercise by increasing their intake on day 2 (Fig. 1(b)). The $48 \mathrm{~h}$ energy balance with the exercise deficit was neither significantly different from zero nor significantly different from that with the diet deficit. However,

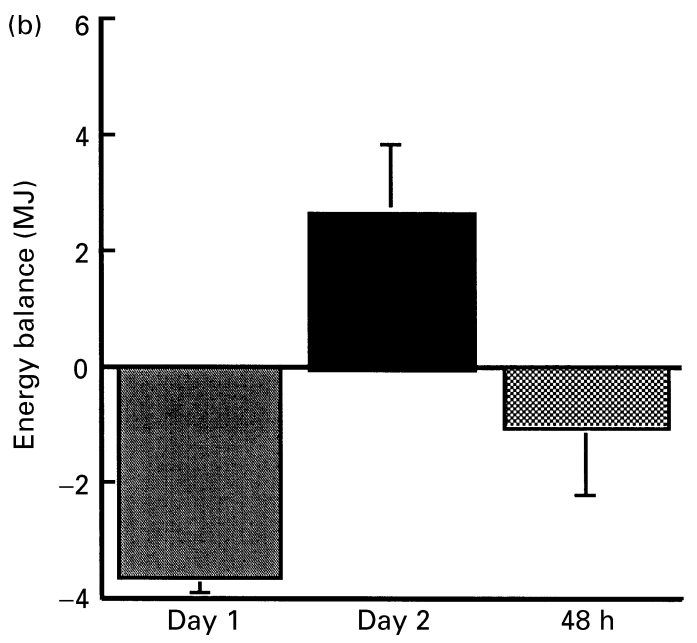

Fig. 1. Energy balance on manipulation day (day 1 ; mIII), outcome day (day $2 ; \boldsymbol{m}$ ) and $48 \mathrm{~h}$ ( $\mathrm{m}$ ) for subjects in an energy deficit by either (a) diet or (b) exercise. Under both conditions $48 \mathrm{~h}$ energy balance was not significantly different from zero. Values are means with their standard errors represented by vertical bars. 
there were large inter-subject variabilities, suggesting that different individuals may have different susceptibilities to obesity. Again, this study indicates that in the face of an energy deficit, created either through diet or exercise, there are powerful homeostatic mechanisms that drive the restoration of energy balance. However, these studies do not indicate the mechanisms which operate with either the diet or exercise deficits.

\section{Intervention studies}

The studies described earlier are highly experimental, and have used lean subjects who were not actively attempting to lose weight. In contrast, most intervention studies have used subjects who are overweight in order to investigate the effects of diet and exercise as part of a voluntary weight-loss programme. The latter studies have shown that it is possible to maintain an energy deficit through diet alone, at least in the short term; however, most weight-control programmes recognize the need for strong cognitive control to override powerful physiological drives to eat. Most studies of diet alone $v$. diet plus exercise show little benefit of an added exercise regimen on weight loss (Svendson et al. 1993; Geliebter et al. 1997), suggesting that there is a physiological compensation for the energy cost of the exercise. This mechanism is supported by a meta-analysis of almost 500 weight-loss interventions in which Miller et al. (1997) showed that there was no significant difference between initial weight loss per week for diet alone $(0.98$ (SE 0.06) kg) or diet plus exercise $(1.00$ (SE 0.06) kg) interventions, or in total weight loss $(10.7(\operatorname{SE} 0.5) \mathrm{kg}$ v. $11.0 \quad(\mathrm{SE} 0 \cdot 6) \mathrm{kg})$. However, some studies do indicate that in the follow-up period, where the aim is to maintain the weight loss, an effect of exercise becomes apparent. For example, Pavlou et al. (1989) showed a marked effect of exercise during the follow-up period when dietary control was relaxed. Subjects who continued to exercise or took up exercise in the followup period were much more likely to maintain their lower body weight than those who undertook no exercise. In their meta-analysis Miller et al. (1997) found weak evidence for the superiority of the diet plus exercise regimen over the diet-only regimen in the follow-up period, in that $77 \%$ of the initial weight loss was maintained in this group compared with $56 \%$ for the diet-only group.

Overall, the evidence suggests that although exercise seems to have only a small role in the attainment of an energy deficit and thus weight loss, it has a stronger effect on the prevention of a positive energy balance and weight regain. In addition, exercise may help to prevent the loss of lean tissue, and thus protect against a fall in BMR.

\section{Energy surplus \\ Epidemiological data}

Data from many large epidemiological studies suggest that sedentary lifestyles are associated with an increased risk of weight gain (Jebb \& Moore, 1999). One of the more comprehensive analyses is that of Coakley et al. (1998). Fig. 2 shows measures of leisure-time physical activity in a cohort of professional males aged 45-54 years. Cross-sectional data from each of the four study years shows that regular exercisers were much less likely to be overweight than those who were regularly inactive. Between 5 and $10 \%$ of those individuals who had maintained high levels of activity were classified as obese compared with between 15 and $24 \%$ of those individuals with low activity levels. Prospectively, those groups of individuals who either undertook regular exercise or who increased their activity showed a smaller increase in the prevalence of obesity than those groups who were either inactive or decreased their activity. For example, between 1990 and 1992 there was a plateau in the prevalence of obesity in the high- and increased-activity groups; however, in the low- and decreased-activity groups prevalence of obesity continued to rise. There was no evidence that exercise led to weight loss, but rather an attenuation of weight gain.

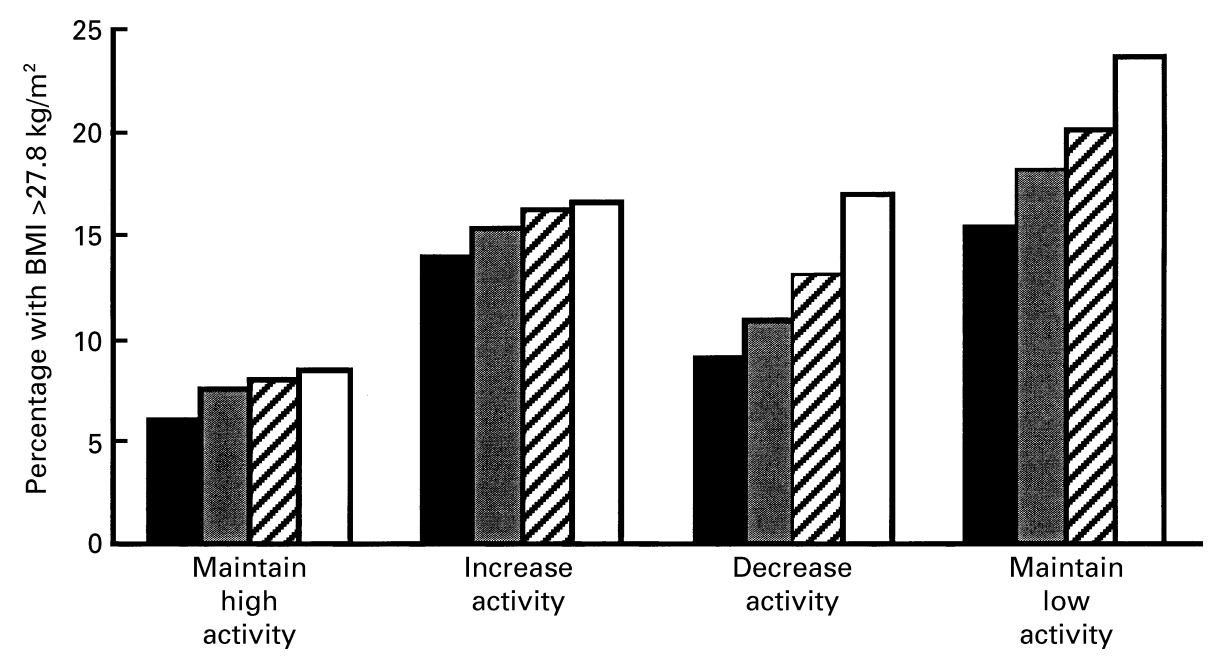

Fig. 2. Prevalence of obesity in men aged 45-54 years with different levels of habitual vigorous activity, based on 3666 males who were non-

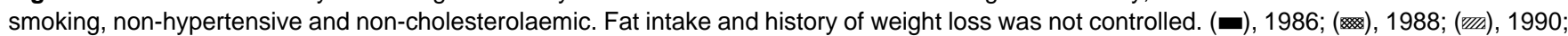
$(\square)$, 1992. (Adapted from Coakley et al. 1998.) 


\section{Coupling between intake and expenditure}

The influence of exercise (mode, intensity, duration etc.) on energy intake and energy balance has been investigated in short-term experimental studies in an attempt to elucidate some of the possible mechanisms involved. In a series of studies, Blundell's group (King \& Blundell, 1995; King et al. 1996, 1997) have shown that in the short term exercise does not increase hunger or appetite, does not increase energy intake and, therefore, can aid weight control primarily due to the energy cost of the exercise per se rather than indirect effects on appetite. In one of their earlier studies (King \& Blundell, 1995), in which they investigated the effects of exercise on subsequent energy intake, lean males who were regular exercisers ate freely from a low-fat or a high-fat diet preceding either a period of rest or a period of exercise. Energy intake, measured both at a test lunch and for the rest of the day, was increased on the high-fat diet relative to the low-fat diet. This finding is consistent with the phenomenon of high-fat hyperphagia, where diets high in fat and consequently of higher energy density encourage overconsumption (Poppitt, 1995). In contrast, exercise had no effect on energy intake with either the high-fat or the low-fat diets, i.e. there was no dietary compensation for the energy cost of the exercise bout. Accordingly, if energy intake is expressed in terms of energy requirements, the exercise regimen results in a reduced energy balance, since the exercise increases energy needs. When the experiment was repeated in lean unrestrained females (King et al. 1996) the results were similar, although there was a nonsignificant tendency for a higher intake after exercise, suggesting that different mechanisms may operate in females compared with males. This potential gender difference has also been suggested from other studies. Staten (1991) measured energy intake on five consecutive sedentary days and compared this intake with that for five consecutive days in which there was a $60 \mathrm{~min}$ exercise session. It was reported that females had similar intakes on both protocols; however, there was a partial energy compensation for the energy cost of the exercise in the males. Contrary to the popular belief that exercise will cause an automatic increase in appetite, most short-term experimental studies have shown that there is no dietary compensation for added exercise. However, this study and the majority of studies have no measures of energy expenditure, thus the status of energy balance can only be estimated. Many studies have also relied on self-reported energy intake, which is often unreliable (Black et al. 1993).

Murgatroyd et al. (1999) have attempted to extend this work using the whole-body calorimeter. Subjects spent two full days in the calorimeter on four different occasions and followed four different protocols. They ate freely from either a high-fat (60\% energy from fat) or a low-fat diet (35 $\%$ energy from fat) and followed either an exercise protocol (additional exercise across the day with an energy cost of approximately $2.5 \mathrm{MJ}$ ) or a sedentary protocol. The $24 \mathrm{~h}$ energy balance did not differ between the first and second day of each visit. Fig. 3 shows that subjects had a lower energy balance on the low-fat diet relative to the high-fat diet which was significant $(P<0.002)$ between the sedentary protocols. The lower energy balance with the low-fat diet

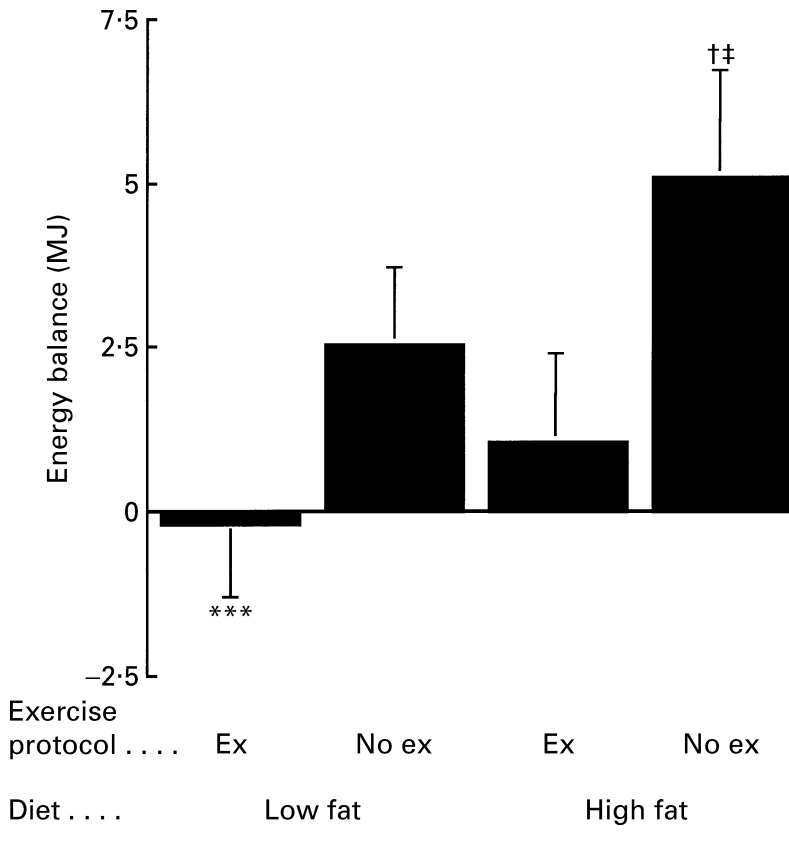

Fig. 3. The $24 \mathrm{~h}$ energy balance for lean males resident in a wholebody calorimeter. Subjects ate freely from either a high-fat or a lowfat diet and followed either a sedentary (no ex) or an exercise (ex) protocol. Values are means with their standard errors represented by vertical bars. Mean value was significantly different from that for lowfat, ex: ${ }^{* * *} P<0.001$. Mean value was significantly different from that for low-fat, no ex: $\dagger P<0.002$. Mean value was significantly different from those for low-fat, ex and high-fat, ex: $\ddagger P<0.0001$. (Adapted from Murgatroyd et al. 1999.)

can be attributed to the energy cost of the exercise bouts; energy intake was similar with both low-fat protocols. However, with the high-fat protocol, the less-positive energy balance cannot be attributed entirely to the energy cost of the exercise. There is clear evidence of an interaction between the energy cost of the exercise and the macronutrient composition of the diet, such that there has been a suppression of the high-fat hyperphagia seen on the nonexercise day. This finding is potentially very important in the light of modern lifestyles which show lowered levels of physical activity and a high proportion of fat in the diet. Lissner et al. (1997) have shown further evidence of an activity-macronutrient interaction. Data from their prospective study indicated that weight changes were only significantly dependent on dietary fat content in sedentary women. This finding, also, implies that physical activity is protective against weight gain in the face of high-fat diets.

A recent study has examined the effects of a $2090 \mathrm{~kJ}$ $(500 \mathrm{kcal})$ or $268 \mathrm{~kJ}(64 \mathrm{kcal})$ breakfast on subsequent energy intake with or without an added exercise period with an energy cost equivalent to approximately $1254 \mathrm{~kJ}$ (300 kcal; Hubert et al. 1998). This protocol effectively created a less-positive energy balance either through diet, exercise, or both in the same group of unrestrained lean females. Energy intake was greater at lunch following the low-energy breakfast relative to the higher-energy breakfast. There were, however, no effects of exercise on energy intake with either dietary regimen. Again, there was no dietary compensation for the exercise, and hence the 
exercise intervention resulted in a reduced energy balance. This finding suggests that the body recognizes the energy deficit created by the reduced energy intake; in contrast, the removal of energy by exercise had not precipitated any compensatory response. This situation suggests that there are different mechanisms operating in response to the two different strategies to create an energy deficit, and would favour exercise as the more successful route to a negative energy balance.

These results contrast with those described previously (Moore et al. 2000), which showed full compensation for an exercise-induced deficit. Possible reasons for this difference include different subjects (gender and habitual-activity status), energy balance, and duration of the experiment. There may also be important cognitive influences which override physiological effects, creating artefactual differences between the two protocols.

Racette et al. (1995) have shown some evidence that exercise deficits are better maintained than dietary deficits. Obese women enrolled in a weight-loss study were randomly assigned to an exercise or a sedentary protocol. Prescribed energy intake was designed such that energy deficits would be similar on both regimens. Compliance with the dietary regimen was better on the exercise protocol than on the sedentary protocol, being exceeded by only 0.7 (SD 1.5) $\mathrm{MJ} / \mathrm{d}$ on the exercise protocol but by 2.3 (SD 1.6) $\mathrm{MJ} / \mathrm{d}$ on the sedentary protocol. Weight loss on the exercise protocol was significantly greater $(10.8$ (SD 3.2) $\mathrm{kg} v .8 \cdot 1$ (SD 2.3) kg; $P=0 \cdot 01$ ).

These studies have shown evidence of a coupling between energy intake and energy expenditure when the restriction is induced through diet. Under the condition of energy surplus removal of energy by exercise seems to be poorly compensated for, suggesting that this coupling between expenditure and intake is weaker. However, there is some discrepancy between different studies, and these differences probably arise from differences in study protocols and the characteristics of the subjects tested.

\section{Summary}

Fig. 4 summarizes our current understanding of the role of diet and physical activity in the regulation of body weight, and highlights the asymmetry in appetite control under conditions of energy deficit and energy surplus. Homeostatic physiological mechanisms conspire to protect the body against energy deficits, and weight loss must usually be achieved through strong cognitive control. Successful weight loss usually involves dietary restriction, although it may also be achieved through increased exercise. Conversely, there seem to be only very weak homeostatic mechanisms to restore energy balance in the face of an energy surplus. It is clear from the rising prevalence of obesity that it is relatively simple to slip into an energy surplus, through the passive overconsumption associated with high-fat diets and modern sedentary lifestyles. There is good evidence that low-fat diets reduce the risk of weight gain through a reduction in the energy density of the diet, and also that exercise attenuates weight gain, although here the precise mechanism is unclear. Some studies have shown

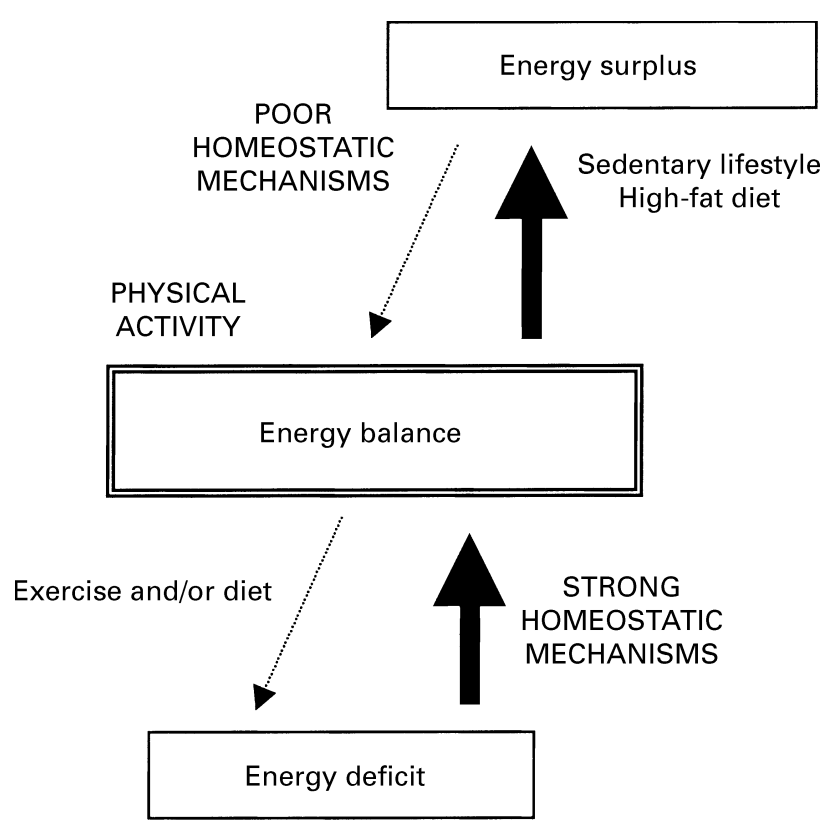

Fig. 4. Asymmetry of body-weight regulation. Energy deficits are difficult to attain, either through a restricted diet or increased exercise. These deficits are opposed by strong physiological mechanisms which restore energy balance. In contrast, it is relatively simple to slip into an energy surplus, and there are only weak mechanisms to restore balance. It is becoming evident that exercise may have a role in the attenuation of a positive energy balance and subsequent weight gain.

an interaction between high-fat diets and inactivity in the aetiology of weight gain, but there is no evidence as yet to identify whether low-fat diets and physical activity may have synergistic effects in terms of weight loss.

However, since the public health benefits of physical activity are now well recognized in terms of the prevention and detection of some prevalent diseases (e.g. hyperlipidaemia, hypertension, diabetes mellitus and cancer; Burnham, 1998), there is every reason to continue to promote an increase in physical activity alongside dietary strategies to tackle the epidemic of obesity.

\section{References}

Black AE, Prentice AM, Goldberg GR, Jebb SA, Bingham SA, Livingstone MB \& Coward WA (1993) Measurements of total energy expenditure provide insights into the validity of dietary measurements of energy intake. Journal of the American Dietetic Association 93, 572-579.

Blundell JE, King NA (1996) Overconsumption as a cause of weight gain: behavioural-physiological interactions in the control of food intake (appetite). Ciba Foundation Symposium 201, 138-154.

Burnham JM (1998) Exercise is medicine: Health benefits of regular physical activity. Journal of the Louisiana State Medical Society 150, 319-323.

Campfield L, Smith F, Gulsez Y, Devos R \& Burn P (1995) Mouse $o b$ protein: evidence for a peripheral signal linking adiposity and central neural networks. Science 269, 546-549.

Coakley EH, Rimm EB, Colditz G, Kawachi I \& Willett W (1998) Predictors of weight change in men: results from the Health Professionals study. International Journal of Obesity 22, 89-96. 
Figlewicz DP, Stein LJ, West D, Porte D Jr \& Woods SC (1986) Intracisternal insulin alters sensitivity to CCK-induced meal suppression in baboons. American Journal of Physiology 250, R856-R860.

Flatt JP (1987) The difference in storage capacities for carbohydrate and for fat, and its implications in the regulation of body weight. Annals of the New York Academy of Sciences 499, 104-123.

Geliebter A, Maher MM, Gerace L, Gutin B, Heymsfield SB \& Hashim SA (1997) Effects of strength or aerobic training on body composition, resting metabolic rate, and peak oxygen consumption in obese dieting subjects. American Journal of Clinical Nutrition 66, 557-563.

Goldberg GR, Murgatroyd PR, McKenna APM, Heavey PM \& Prentice AM (1998) Dietary compensation in response to a covert imposition of negative energy balance by removal of fat or carbohydrate. British Journal of Nutrition 80, 141-147.

Hubert P, King NA \& Blundell JE (1998) Uncoupling the effects of energy expenditure and energy intake: Appetite response to short-term energy deficit induced by meal omission and physical activity. Appetite 31, 9-19.

Jebb SA \& Moore MS (1999) Contribution of a sedentary lifestyle and inactivity to the etiology of overweight and obesity: current evidence and research issues. Medicine and Science in Sports and Exercise 31, S534-S541.

Kennedy GC (1953) The role of depot fat in the hypothalamic control of food intake in the rat. Proceedings of the Royal Society 140B 579-592.

King NA \& Blundell JE (1995) High fat foods overcome the energy expenditure induced by high intensity cycling or running. European Journal of Clinical Nutrition 49, 114-123.

King NA, Lluch A, Stubbs RJ \& Blundell JE (1997) High dose exercise does not increase hunger or energy intake in free-living males. European Journal of Clinical Nutrition 51, 478-483.

King NA, Snell L, Smith RD \& Blundell JE (1996) Effects of shortterm exercise on appetite response in unrestrained females. European Journal of Clinical Nutrition 50, 663-667.

Lissner L, Heitmann BL \& Bengtsson C (1997) Low fat diets may prevent weight gain in sedentary women: prospective observations from the population study of women in Gothenburg, Sweden. Obesity Research 5, 43-48.

Mayer J (1953) Glucostatic mechanism of regulation of food intake. New England Journal of Medicine 249, 13-16.

Miller WC, Koceja DM \& Hamilton EJ (1997) A meta-analysis of the past 25 years of weight loss research using diet, exercise or diet plus exercise intervention. International Journal of Obesity 21, 941-947.
Ministry of Agriculture, Fisheries and Foods (1991) Household Food Consumption and Expenditure 1940-94. London: H.M. Stationery Office.

Monteiro CA, Mondini L, de Souza AL \& Popkin BM (1995) The nutrition transition in Brazil. European Journal of Clinical Nutrition 49, 105-113.

Moore MS, Murgatroyd PR, Goldberg GR \& Jebb SA (2000) Restoration of energy balance following a diet- or exerciseinduced energy deficit. Proceedings of the Nutrition Society (In the Press).

Murgatroyd PR, Goldberg GR, Leahy FE, Gilsenan MB \& Prentice AM (1999) Effects of inactivity and diet composition on human energy balance. International Journal of Obesity 23, $1269-1275$.

Pavlou K, Krey S \& Steffee W (1989) Exercise as an adjunct to weight loss and maintenance in moderately obese subjects. American Journal of Clinical Nutrition 49, 1115-1123.

Popkin BM \& Doak CM (1998) The obesity epidemic is a worldwide phenomenon. Nutrition Reviews 56, 106-114.

Poppitt SD (1995) Energy density of diets and obesity. International Journal of Obesity 19, Suppl. 5, S20-S26.

Prentice AM \& Jebb SA (1995) Obesity in Britain: gluttony or sloth? British Medical Journal 311, 437-439.

Prescott-Clarke P \& Primatesta P (1999) Health Survey for England 1997. London: The Stationery Office.

Racette SB, Scholler DA, Kushner RF \& Neil KM (1995) Exercise enhances dietary complience during moderate energy restriction in obese women. American Journal of Clinical Nutrition $\mathbf{6 2}$ 345-349.

Schwartz MW, Baskin DG, Kaiyala KJ \& Woods SC (1999) Model for the regulation of energy balance and adiposity by the central nervous system. American Journal of Clinical Nutrition 69, 584-596.

Seidell JC (1999) Obesity: a growing problem. Acta Paediatrica Scandinavica 428, Suppl., 46-50.

Staten MA (1991) The effect of exercise on food intake in men and women. American Journal of Clinical Nutrition 53, 27-31.

Svendson OL, Hassager L \& Christiansen C (1993) Effect of energy-restrictive diet, with or without exercise, on lean tissue mass, resting metabolic rate, cardiovascular risk factors and bone in overweight postmenopausal women. American Journal of Medicine 95, 131-140.

Weinsier RL, Hunter GR, Heini AF, Goran MI \& Sell SM (1998) The etiology of obesity: relative contribution of metabolic factors, diet, and physical activity. American Journal of Medicine 105, 145-150.

Woods SC, Stein LJ, McKay LD \& Porte D Jr (1979) Chronic intracerebroventricular infusion of insulin reduces food intake and body weight of baboons. Nature 282, 503-505. 